\title{
Planejamento e avaliação institucional: um indicador do instrumento de avaliação do SINAES
}

\author{
Enedina Betânia Leite de Lucena Pires Nunes \\ Michelle Matilde Semigueem Lima Trombini Duarte \\ Isabel Cristina Auler Pereira
}

Resumo: Este artigo reflete sobre a dimensão "Planejamento e Avaliação Institucional" abordada pela Lei 10.861/2004, que implementa o Sistema Nacional de Avaliação da Educação Superior (Sinaes), o qual contribui para evidenciar a interação da avaliação interna no planejamento da Universidade Federal do Tocantins (UFT) e suas atividades acadêmicas, de forma a demonstrar a evolução da instituição e a busca da melhoria da qualidade. O referencial teórico possibilitou refletir sobre a avaliação e o planejamento como subsídios para a melhoria e aperfeiçoamento das instituições, baseou-se em teóricos que tratam sobre a temática, e na regulamentação da avaliação do ensino superior no Brasil a partir do Sistema Nacional de Avaliação da Educação Superior (Sinaes), criado em 2004; de documentos institucionais e oficiais. Trata-se de pesquisa qualitativa com análise documental e bibliográfica que demonstra que a avaliação institucional articulada ao planejamento pode ser utilizada como uma valiosa ferramenta na busca constante por melhorias e por soluções às fragilidades apontadas pelo planejamento e pela própria avaliação da instituição, podendo subsidiar a gestão na tarefa de implementar ações sustentáveis.

Palavras-chave: Avaliação externa. Avaliação interna. SINAES.

\section{Planning and institutional assessment: an assessment tool indicator of SINAES}

Abstract: This article reflects on the "Planning and Institutional Assessment" element addressed by Law 10.861/2004, which implements the National System of Higher Education Assessment (Sinaes). It contributes to highlighting the interaction of internal assessment in the planning at the Federal University of Tocantins (UFT) and its academic activities in order to demonstrate the development of the institution and its pursuit of quality improvement. The theoretical framework enabled the reflection of the evaluation and planning in aiding the improvement and enhancement of the institutions. This framework was based on the work of such theorists on the subject and on the regulation of evaluation of higher education in Brazil, starting from Sinaes, created in 2004; institutional and official documents. This article is a qualitative research paper with documentary and bibliographical analysis which shows that the institutional evaluation of planning can be used as a valuable tool in the constant search for improvements and for solutions to the weaknesses identified by the planning and by the assessment of the institution, and may assist the management in the task of implementing sustainable actions.

Key words: External evaluation. Internal evaluation. SINAES. 


\section{Introdução}

O presente artigo busca aprofundar a reflexão sobre a dimensão "Planejamento e avaliação institucional" abordada pela Lei 10.861, de 2004, que instituiu o Sinaes, inserida no Instrumento de Avaliação Externa, e que contribui para evidenciar a interação da avaliação interna no planejamento da Universidade Federal do Tocantins (UFT) e suas atividades acadêmicas, de forma a demonstrar a evolução da instituição e a busca da melhoria da qualidade. O foco desta dimensão é a evolução acadêmica, a descrição e a identificação dos principais elementos do processo avaliativo da IES (interno e externo) em relação ao Plano de Desenvolvimento Institucional (PDI), incluindo os relatórios elaborados pela Comissão Própria de Avaliação (CPA), da Universidade Federal do Tocantins (UFT).

Este estudo surgiu a partir do envolvimento e experiência de pesquisadoras nas atividades universitárias e da constatação de que a avaliação institucional é um instrumento de planejamento e de ações acadêmico-administrativas de melhoria institucional. O processo de discussão e elaboração do Plano de Desenvolvimento Institucional (PDI) na UFT foi conduzido pela Pró-Reitoria de Avaliação e Planejamento (Proap), no ano de 2015, tendo como base os trabalhos oriundos do Planejamento Estratégico (PE) do período de 2014 a 2022. O PE foi elaborado de forma conjunta com a Reitoria e os sete câmpus universitários, por meio de comissões de planejamento, utilizando-se de metodologia que previa encontros com professores, técnicos, estudantes e comunidade externa, momento em que foram expostas questões relativas às realidades de cada câmpus. A partir dos dados coletados, foi elaborado um mapa situacional com a apresentação de modelos desenhados com vistas a subsidiar os Planos de Desenvolvimento do Câmpus (PDC) e as ações táticas da matriz, que serviram de base para a construção do Plano de Desenvolvimento Institucional (PDI) para os anos de 2016-2020.

A avaliação institucional constitui-se em ferramenta fundamental que possibilita perceber os erros e equívocos e a partir daí vencer os obstáculos, promovendo, dessa forma, o crescimento da instituição e da comunidade acadêmica envolvida. Articulada ao planejamento, pode se constituir em uma ação efetiva que assegure o desenvolvimento de uma instituição de ensino superior.

Nesta pesquisa, apresentamos um breve histórico sobre a avaliação institucional no Brasil, chegando ao atual Sistema Nacional de Avaliação da Educação Superior (Sinaes) que integra os processos avaliativos das instituições de ensino superior, e, posteriormente, ao processo de discussão e elaboração do Plano de Desenvolvimento Institucional da Universidade Federal do Tocantins. Foram utilizados estudos e citações de autores que tratam do tema como Dias Sobrinho (2003, 2005), Gatti (2000) e Ristoff (2003). O processo de construção da Avaliação na UFT foi iniciado em outubro de 2003, ano de sua implantação, passando a se constituir como um instrumento de aperfeiçoamento do desempenho de suas funções universitárias e da gestão acadêmica na busca da melhoria da qualidade das suas atividades de ensino, de pesquisa e de extensão.

Este estudo é relevante, pois significa pensar a universidade sob um de seus alicerces que é a avaliação institucional, ferramenta importante na busca constante por melhoria da qualidade do ensino, da pesquisa e da extensão, à medida que possa subsidiar os gestores na tomada de decisão para implementar ações sustentáveis. 


\section{0 sistema nacional de avaliação da educação superior (SINAES) no contexto do ensino superior}

O sistema educacional brasileiro vem passando por importantes reformulações nas políticas de avaliação do ensino superior, tornando-se eminente a necessidade de acompanhamento mais efetivo do planejamento e das ações implementadas pelas instituições. O Sistema Nacional de Avaliação da Educação Superior (Sinaes) possui uma série de instrumentos como a autoavaliação, a avaliação externa, o Exame Nacional de Desempenho dos Estudantes (Enade), a avaliação dos cursos de graduação e os instrumentos de informação (Censo e cadastro), que serão discutidos ao longo do texto. Os resultados dessas avaliações possibilitam traçar um panorama da qualidade dos cursos e instituições de educação superior do país, sendo os dados obtidos utilizados para orientação institucional e efetividade acadêmica e social; pelos órgãos governamentais para orientar políticas públicas; e pelos estudantes, pais de alunos, instituições acadêmicas e público em geral para orientar suas decisões quanto à realidade dos cursos e das instituições. Podem ainda contribuir para a formulação de políticas institucionais, possibilitando a articulação do planejamento e avaliação, e, consequentemente, a melhoria do desenvolvimento institucional.

A expansão do número de instituições e cursos ocorrida a partir da última década deste século amplia a importância do Sistema de Avaliação da Educação Superior, fazendo com que as instituições busquem formas de viabilizar, ampliar e melhorar as suas políticas, tais como investimentos na qualificação docente e na infraestrutura física e organizacional. Para acompanhar as mudanças e promover as melhorias, as instituições educacionais, tanto públicas como privadas, têm promovido o processo de avaliação institucional, demonstrando melhor compreensão da importância do papel da avaliação institucional, não sendo apenas para cumprir a legislação.

A partir dessa regulamentação, as instituições de ensino superior devem definir o seu Plano de Desenvolvimento Institucional (PDI) e a política institucional de avaliação institucional, delimitando as ações e programas previstos no Instrumento de Avaliação Institucional Externa, do Ministério da Educação, que subsidia os atos de credenciamento, recredenciamento e transformação da organização acadêmica (BRASIL, 2014a, 2014b). O grande desafio das instituições de ensino superior é fazer do processo avaliativo uma ferramenta eficiente e eficaz, porém não imediatista ou circunstancial, proporcionando maior eficiência no processo de ensino e na gestão universitária.

\subsection{Fundamentos da avaliação institucional}

A avaliação para muitos tem um caráter punitivo, principalmente nas instituições educacionais, e isso leva ao temor de ser avaliado, de não gostar de ser avaliado. Segundo Gatti (2000, p. 94), "avaliações são processos que devem gerar estímulo, servir de alavanca, servir à mudança e à transformação e não serem utilizados para rebaixamento de autoestima, seletividade, punição, diminuição de valia".

Imbuído dessa perspectiva, o Sistema Nacional de Avaliação da Educação Superior (SINAES), em conformidade com a Lei 10.861/2004, avalia as dimensões do ensino, pesqui- 
sa, extensão, responsabilidade social, gestão da instituição e corpo docente, verificando a coerência destas com as diretrizes estabelecidas no Plano de Desenvolvimento Institucional (PDI). De acordo com o disposto no $\S^{\circ}$, art. $1^{\circ}$, da referida Lei:

\begin{abstract}
O SINAES tem por finalidades a melhoria da qualidade da educação superior, a orientação da expansão da sua oferta, o aumento permanente da sua eficácia institucional e efetividade acadêmica e social e, especialmente, a promoção do aprofundamento dos compromissos e responsabilidades sociais das instituições de educação superior, por meio da valorização de sua missão pública, da promoção dos valores democráticos, do respeito à diferença e à diversidade, da afirmação da autonomia e da identidade institucional (BRASIL, 2004, p. 3).
\end{abstract}

É constituído de três processos específicos: avaliação dos estudantes por meio do Enade, avaliação dos cursos e avaliação institucional (interna e externa), além dos instrumentos de informação: censo da educação superior e cadastro de instituições e cursos. O primeiro processo é o Exame Nacional de Desempenho dos Estudantes (ENADE), instituído pela Portaria 40/2010, Art. 33-D, que estabelece o objetivo de aferir "o desempenho dos estudantes em relação aos conteúdos programáticos previstos nas diretrizes curriculares do respectivo curso de graduação, e as habilidades e competências adquiridas em sua formação" (BRASIL, 2010 , p. 23). Tem como instrumentos básicos a prova, o questionário dos estudantes e do coordenador do curso. O segundo processo se refere à Avaliação dos cursos de graduação realizada por comissões externas de avaliadores designadas pelo Instituto Nacional de Estudos e Pesquisas Educacionais Anísio Teixeira (INEP), para verificação das condições de ensino relativas ao perfil do corpo docente, infraestrutura e organização didático-pedagógica. E o terceiro processo se refere à Avaliação Institucional (interna e externa) como um dos componentes do Sinaes.

A Avaliação Institucional está relacionada à melhoria da qualidade da educação superior; à orientação da expansão de sua oferta; à efetividade acadêmica e social; ao aprofundamento dos compromissos e responsabilidades sociais das instituições de educação superior por meio da valorização de sua missão pública, da promoção dos valores democráticos, do respeito à diferença e à diversidade, da afirmação da autonomia e da identidade institucional e se divide em duas modalidades: 1) a autoavaliação, coordenada pela Comissão Própria de Avaliação (CPA) e orientada pelas diretrizes e pelo roteiro de autoavaliação elaborado pelo Inep e pela Comissão Nacional de Avaliação da Educação Superior (CONAES); 2) a avaliação externa, que tem como referência os padrões de qualidade para a educação superior, expressos nos instrumentos de avaliação, e nos relatórios das autoavaliações (BRASIL, 2004).

A autoavaliação é coordenada pela CPA e contempla a análise global e integrada das dez dimensões organizadas em cinco eixos previstos no Instrumento de Avaliação Institucional Externa: Eixo 1 - Planejamento e Avaliação Institucional: considera a dimensão 8 (Planejamento e Avaliação) do Sinaes; Eixo 2 - Desenvolvimento Institucional; Eixo 3 - Políticas Acadêmicas; Eixo 4 - Políticas de Gestão; e Eixo 5 - Infraestrutura Física (BRASIL, 2014). Além desses eixos, as instituições devem considerar as três dimensões: organização didáticopedagógica, corpo docente e instalações físicas, adotadas no Instrumento de Avaliação dos cursos de graduação presencial e a distância. Como estratégia para as avaliações internas (institucional e de cursos) poderão ser adotados os mesmos indicadores dos instrumentos de ava- 
liação in loco do Inep com vistas à melhoria contínua da qualidade dos serviços prestados para uma avaliação externa efetiva.

É relevante haver coerência entre o planejamento e a avaliação externa e a avaliação interna (autoavaliação) no sentido de auxiliar a instituição a identificar suas potencialidades e fragilidades, carências e necessidades, a definir suas prioridades, contribuindo para a sua evolução a partir dos processos de planejamento e avaliação institucional como instrumento de gestão e de ações acadêmicas e administrativas de melhoria institucional junto à comunidade acadêmica e à sociedade.

\section{Sinalizadores e indicadores de avaliação: algumas reflexões}

O uso de indicadores do Sinaes nos processos regulatórios avaliativos das instituições de ensino superior serve de referencial básico para a avaliação externa como também como subsídio para a autoavaliação institucional. Com o propósito de auxiliar na efetivação da proposta de avaliação institucional, Dias e Ketzer (2007) apresentam um conjunto de diferentes sinalizadores e indicadores visando a potencializar a construção de indicadores, levando-se em conta aspectos ligados ao Projeto Pedagógico (PP), aos recursos humanos, à infraestrutura e à gestão da graduação. Destacam-se os seguintes indicadores para cada um dos aspectos referenciados pelas autoras.

a) Projeto Pedagógico (PP): ações internas e externas com participação em Conselhos Comunitários; articulação ensino, pesquisa e extensão; coerência entre missão, diretrizes, políticas, metas e objetivos da IES e o explicitado em seu PP; envolvimento do corpo docente, discente e técnico administrativo; educação continuada; estudos interdisciplinares; formação humanística; condições de acesso para portadores de necessidades especiais; metodologias de ensino; estágios; organização curricular; políticas de ensino, pesquisa e extensão coerentes com o plano de desenvolvimento institucional; relação do projeto pedagógico do curso com o projeto pedagógico institucional; relevância e responsabilidade social; entre outros.

\section{b) Recursos humanos:}

Corpo docente: compromisso com a IES; ética; habilidades didático-pedagógicas; trabalho em grupos de estudo, núcleos de pesquisa, programas sociais e comunitários; produção acadêmica (científica, artística, cultural e tecnológica); produção acadêmica; qualificação profissional; avaliação docente e discente; atendimento extraclasse; relacionamento professoraluno e interpessoal; titulação; entre outros.

Corpo técnico administrativo: dedicação; efetividade; formação continuada; iniciativa; qualificação profissional; relacionamento interpessoal; trabalho em grupo; entre outros.

Corpo discente: acesso; acompanhamento de egressos; criatividade; diplomação; ética; evasão; iniciativa; resolução de problemas; trabalho em grupo; desenvolvimento sociocultural; domínio de línguas; participação em programas de iniciação científica, à docência, monitorias, eventos, mobilidade; produção acadêmica; retenção; uso de internet, bibliotecas, laboratórios; entre outros.

c) Infraestrutura: acesso aos portadores de necessidades especiais; bibliotecas; mobiliários e equipamentos; equipamentos de informática; hospital universitário; laboratórios; órgãos complementares; instalações administrativas e acadêmicas, salas de aula; entre outros. 
d) Gestão: acesso e fluxo de informação; ambiente externo; convênios; custos; fomento; oferta de cursos; intercâmbios; participação nos processos deliberativos; planejamento; plano de desenvolvimento institucional; processos de avaliação; programas de apoio ao estudante; sistema gerencial e de informação, sustentabilidade financeira; entre outros.

Além dos indicadores elencados, a Instituição, em seu programa de avaliação articulado ao Plano de Desenvolvimento Institucional, deve contemplar as seguintes dimensões, segundo Dias e Ketzer (2007, p. 93):

Global: isto é, o reconhecimento das demandas sociais e científicas, bem como as diretrizes contidas na LDB, Constituição Federal e Plano Nacional de Educação.

Específica: no que se refere aos princípios e diretrizes contidas no Plano Nacional de Graduação (oferta de ensino superior e sua expansão, formação de professores, diretrizes curriculares, sistema de avaliação, qualificação de professores, educação à distância, financiamento e fomento à produção científica).

Particular: diz respeito à vocação e inserção regional da IES, isto é, a garantia da sua especificidade, da sua história e das diferenças culturais, dos vínculos e compromissos estabelecidos com a comunidade regional. Os indicadores arrolados para avaliar tal dimensão não podem ser desconsiderados pela avaliação externa. Os avaliadores externos deverão apreciar e reconhecer os julgamentos apreciativos contidos no relatório da Instituição, avaliando a relação entre os resultados obtidos e as metas e ações desenvolvidas no seu Plano de Desenvolvimento Institucional.

Sugere-se, tomando como base esses indicadores e dimensões, que a Instituição possa ter uma sustentação para o seu planejamento e avaliação institucional, em complementariedade ao processo de avaliação externa e interna vivenciado no ambiente acadêmico, com vistas ao cumprimento da sua missão e da consolidação da sua visão de futuro.

\section{Dimensão "Planejamento e Avaliação Institucional" no instrumento de Avaliação Ins- titucional Externa}

Por meio da Portaria n ${ }^{\circ}$ 92, de 31 de janeiro de 2014, o Ministério da Educação estabeleceu os indicadores dos eixos das dimensões do Sinaes do Instrumento de Avaliação Externa (BRASIL, 2014b). O Eixo 1 do Instrumento foca o Planejamento e Avaliação e a descrição e a identificação dos principais elementos do processo avaliativo da IES em relação ao PDI, aos relatórios elaborados pela CPA e aos demais documentos institucionais avaliativos do período que constitui o objeto de avaliação (BRASIL, 2014a). Contempla os seguintes indicadores de Planejamento e Avaliação Institucional:

1.1. Evolução institucional a partir dos processos de Planejamento e Avaliação Institucional.

1.2. Projeto/processo de autoavaliação institucional.

1.3. Autoavaliação institucional: participação da comunidade acadêmica.

1.4. Autoavaliação institucional e avaliações externas: análise e divulgação dos resultados.

1.5. Elaboração do relatório de autoavaliação (BRASIL, 2014b, p. 5-6).

Observa-se pelo exposto o estabelecimento de indicadores que fazem com que as instituições demonstrem a evolução institucional em relação ao processo de Planejamento e Avaliação Institucional implantado de maneira a atender às necessidades institucionais, como instrumento de gestão e de ações acadêmico-administrativas de melhoria institucional com parti- 
cipação efetiva da comunidade acadêmica, apresentando resultados, análises, reflexões e proposições de forma a subsidiar planejamento e ações.

Os itens a serem avaliados na dimensão de Planejamento e Avaliação Institucional potencializam a importância da avaliação institucional, relacionando-a ao planejamento, considerando a indissociabilidade entre ensino, pesquisa, extensão e gestão. Esta dimensão é considerada especialmente no que se refere à evolução institucional a partir dos processos de planejamento e avaliação, de forma a atender às necessidades institucionais, como instrumento de gestão e de ações acadêmicas e administrativas de melhoria institucional, e apresentar resultados, análises, reflexões e proposições para subsidiar o planejamento e ações.

Ao elaborar o Plano de Desenvolvimento Institucional (PDI), a instituição deve delimitar na sua política institucional as ações e programas a serem desenvolvidos, sendo recomendável que em seu programa de avaliação institucional estejam definidos os indicadores planejamento e avaliação na condução da referida política. Esta dimensão apresenta indicadores que, ao serem aplicados, deverão ser verificados os processos avaliativos internos e externos da IES e a síntese histórica do planejamento de ações acadêmico-administrativas decorrentes dos resultados das avaliações, além das informações originadas a partir dos relatórios de avaliação interna e externa no âmbito da autoavaliação e das demais avaliações externas representadas nas diversas ações institucionais decorrentes. Desta forma, percebe-se que o instrumento de avaliação evidencia indicadores com critérios de análise subjetivos, porém as instituições são levadas a organizar as informações e os documentos para atender de forma objetiva aos indicadores.

\section{Processo de Planejamento e Avaliação Institucional na UFT}

A área do planejamento e avaliação da Universidade Federal do Tocantins (UFT) está sob a responsabilidade da Pró-Reitoria de Avaliação e Planejamento (Proap), que conduziu no ano de 2015 a construção do Plano de Desenvolvimento Institucional (PDI) tendo como base os trabalhos oriundos do Planejamento Estratégico (PE). O Plano de Desenvolvimento Institucional deve ser pensado para o período mínimo de cinco anos e construído por meio de extenso processo envolvendo representantes de toda a comunidade acadêmica. Para acompanhamento das metas do PDI e alcance de objetivos e indicadores, a Proap pretende realizar periodicamente Reuniões de Avaliação (RA's) com a participação de dirigentes e responsáveis pelas unidades gestoras que apresentarão o andamento das ações e metas e os respectivos resultados alcançados. Um dos objetivos estratégicos seguido pela Proap é instituir a cultura do planejamento e da avaliação, de modo que os instrumentos que tornam o trabalho burocrático deixem de ser meramente mecânicos e legais. Além do PDI e dos Planos de Desenvolvimento dos Câmpus, a UFT tem atuado, na perspectiva de planejamento em curto prazo, com os Planos Anuais de Trabalho (PAT) de cada unidade gestora, compatíveis com o dimensionamento acadêmico e com o Plano de Distribuição Orçamentária (PDO) anual da Universidade.

Este modelo de planejamento adotado pela UFT trabalha os eixos referenciais instituídos no Sistema Nacional de Avaliação da Educação Superior (Sinaes) e propõe um sistema integrado de gestão com base em indicadores de resultados, de forma a contribuir para o de- 
senvolvimento da visão sistêmica, maior articulação entre ações de ensino, pesquisa e extensão, promovendo necessários avanços no modelo de gestão e avaliação institucional.

Para as diretrizes de ação deste processo de planejamento, utilizou-se como instrumentos norteadores o Instrumento de Avaliação Institucional Externa (BRASIL, 2014a) e o Instrumento de Avaliação de cursos de graduação presencial e a distância (BRASIL, 2015), que serviram de balizamento à definição das metas e ações anuais de cada unidade (Pró-reitorias e Câmpus), de acordo com os cinco eixos do Sinaes, traduzindo-se em Planos Anuais de Trabalho (PAT). Dentre os meses de agosto a dezembro de 2015, foram desenvolvidas etapas como levantamento de dados institucionais para subsidiar a proposta, encaminhamento das propostas de ação às unidades; acompanhamento do trabalho das unidades, recebimento e consolidação dos dados; compatibilização e devolução das propostas às unidades.

Toda a concepção do Plano de Desenvolvimento Institucional (PDI) e dos Planos de Desenvolvimento dos Câmpus (PDC) teve por objetivo atender aos requisitos legais do Sinaes e aperfeiçoar seu próprio modelo de planejamento por meio da adoção de uma metodologia participativa de elaboração, como também da implantação de um processo sistemático de acompanhamento e avaliação.

A pesquisa ora desenvolvida reforça que a autoavaliação precisa ser articulada com o planejamento e deve servir, principalmente, como instrumento de gestão na melhoria da instituição, por isso é importante se ter uma avaliação em sintonia, tanto externa como internamente, integrando todos os setores que compõem a universidade. Neste sentido, a Comissão Própria de Avaliação (CPA) da UFT articula-se com as Pró-Reitorias no que concerne à avaliação da instituição, principalmente com a Pró-Reitoria de Avaliação e Planejamento (Proap), e com as coordenações de curso e Pró-Reitoria de Graduação (Prograd) quanto à avaliação de cursos, para produzir subsídios para o planejamento, avaliação e desenvolvimento da instituição e de seus cursos de graduação.

Dias Sobrinho (2005) sinaliza a avaliação como uma dimensão imprescindível da Universidade. Para ele (p. 33), a instituição

precisa saber, de forma permanente e integrada, quais são os valores dominantes nas suas atividades de ensino, pesquisa e extensão e nas suas práticas administrativas. [...] é um exercício com forte sentido pedagógico que não só permite rediscutir os projetos e prioridades essenciais da universidade, suas relações com a ciência, a tecnologia, as letras e as artes e suas interações com a sociedade, como também contribuir para a elaboração mais consistente dos diversos sentidos da instituição.

Complementando, o autor afirma que a avaliação "deve ser uma atividade sistemática e permanente que resulte em uma compreensão global e integrada da Universidade, [...] de todos os aspectos e setores científicos, pedagógicos, políticos e administrativos" (p. 33-34).

Nesse sentido, a avaliação deve ser um processo sistematizado, permanente, contínuo e integrador por envolver múltiplas e complexas tarefas, constituindo-se como instrumento da melhoria da qualidade institucional em todos os seus aspectos. Essa integração das ações do planejamento com a avaliação (externa e interna) permite verificar a efetivação dos indicadores de qualidade da educação superior nos três segmentos: ensino, pesquisa e extensão da UFT. O processo de planejamento e avaliação institucional coordenado pela CPA levará à 
consolidação de uma cultura avaliativa, sendo fundamental para o cumprimento da missão, objetivos e metas da instituição, visando sempre à melhoria da qualidade do ensino oferecido.

No que tange à avaliação, o processo de construção da Avaliação Institucional da UFT se iniciou em outubro de 2003 com a Comissão Central de Avaliação Institucional (CCA), que passou a se chamar Comissão Própria de Avaliação (CPA), fundamentando-se em princípios que garantem participação da comunidade acadêmica, visão de unidade, pluralidade, respeito as suas características e a sua identidade institucional, entre outros.

Para Ristoff (2003, p. 19), “[...] avaliação é, na verdade, uma questão mais complexa do que normalmente se imagina”. Afinal, julgar é difícil e não é este o objetivo deste estudo, mas o de analisar o processo da avaliação institucional na busca da qualidade em uma instituição de educação superior. Neste sentido, a Proap, no ano de 2015, atualizou o Plano de Desenvolvimento Institucional (PDI) para os anos de 2016-2020, que buscará ser um parâmetro para a avaliação institucional e materialização em ações.

De maneira intensa, houve a participação de toda a comunidade acadêmica na construção desse documento, com a realização de oficinas em todos os câmpus, apresentação das diretrizes do Plano nos Conselhos Diretores e suporte intenso da Pró-Reitoria de Avaliação e Planejamento (PROAP) para a composição das ações dos câmpus universitários. Importante destacar que esse Plano de Desenvolvimento Institucional (PDI) foi constituído a partir das ações dos câmpus, que elaboraram seus Planos de Desenvolvimento do Câmpus (PDC), os quais apresentam os objetivos estratégicos, indicadores de desempenho e metas que tornam a estratégia explícita o suficiente, de modo que todas as pessoas envolvidas com o processo possam compreendê-la.

Com a edição desses Planos (PDI e PDC), em 2016, será dada continuidade ao trabalho de envolvimento de todos para que as ações expressas no planejamento sejam bem executadas e tragam resultados efetivamente positivos para a instituição. Com base no exposto, evidencia-se a importância da relação entre a dimensão Planejamento e Avaliação Institucional e o PDI, visto que segundo Cavalieri, Macedo-Soares e Thiollent (2004):

\begin{abstract}
Uma avaliação internalizada pode gerar informações para servir de base de apoio para o planejamento de médio ou longo prazos. Avaliações bem concebidas e realizadas com a participação ativa das comunidades envolvidas podem fornecer relevantes subsídios para diferentes modalidades de planejamento, em particular quando se trata de redesenhar o futuro de determinadas atividades universitárias (CAVALIERI; MACEDO-SOARES; THIOLLENT, 2004, p. 98).
\end{abstract}

Assim, consolida-se a avaliação institucional como um instrumento de gestão, assim como permite à Universidade produzir conhecimentos, identificar os problemas e deficiências, evoluir pedagogicamente em sua atuação junto à comunidade interna e externa, julgando o seu compromisso social e relevância científica e prestando contas à sociedade. Segundo o Sinaes (2009), essas são as funções mais importantes da autoavaliação:

produzir conhecimentos, pôr em questão a realização das finalidades essenciais, identificar as causalidades dos problemas e deficiências, aumentar a consciência pedagógica e a capacidade profissional dos professores, tornar mais efetiva a vinculação da instituição com o entorno social e a comunidade mais ampla, julgar acerca da relevância científica e social de suas ativida- 
des e seus produtos, prestar contas à sociedade, justificar publicamente sua existência e fornecer todas as informações que sejam necessárias ao conhecimento do Estado e da população [...] (p. 106).

Essa reflexão contribui para o amadurecimento da cultura avaliativa institucional articulada ao planejamento, visto que a "universidade é compreendida como um conjunto de processos e relações que se produzem em seu cotidiano" (DIAS SOBRINHO, 2005, p. 15). Percebe-se, assim, que a avaliação institucional se constitui em um processo necessário ao cotidiano da universidade.

\section{Considerações Finais}

A busca pela qualidade na instituição pressupõe um compromisso de autoavaliação permanente e exige uma prática avaliativa articuladora dos processos administrativos e pedagógicos, por meio de uma análise sistemática dos resultados obtidos, no sentido de manter a excelência acadêmica e o aperfeiçoamento da instituição.

A avaliação não pode ser meramente uma ação mecânica para cumprir uma exigência legal, mas um processo sistemático de acompanhamento permanente das instituições de ensino superior em que todos os envolvidos com o processo se aculturem na busca da melhoria da educação superior.

É necessário, entretanto, o amadurecimento de uma cultura de avaliação por parte da comunidade universitária. Há que se entender que a avaliação não é modismo, mas uma prática que deve somar esforços de todos os gestores e colaboradores no sentido de identificar os aspectos mais vulneráveis e que devem ser melhorados na instituição.

Enfim, como promotora do conhecimento, é competência da instituição de ensino superior propiciar mudanças contínuas em prol da sociedade e da comunidade acadêmica, a fim de que se tenha uma melhor qualidade do ensino ofertado e, consequentemente, de vida.

\section{Referências}

BRASIL. Lei no 10.861/2004 - Sistema Nacional de Avaliação da Educação Superior SINAES. Diário Oficial da União, Brasília, 15 abr. 2004. Seção 1. Brasília, 2004.

BRASIL. Ministério da Educação. Instituto Nacional de Estudos e Pesquisas Educacionais Anísio Teixeira. Comissão Nacional de Avaliação da Educação Superior. Sistema Nacional de Avaliação da Educação Superior. Orientações Gerais para o Roteiro da Auto-avaliação das Instituições. Brasília, DF, 2004. Disponível em: <http://download.inep.gov.br/download/superior/sinaes/orientacoes_sinaes.pdf > . Acesso em 22 mar. 2016.

BRASIL. Ministério da Educação. Portaria Normativa nº 40, de 12 de dezembro de 2007. Institui o e-MEC, sistema eletrônico de fluxo de trabalho e gerenciamento de informações relativas aos processos de regulação, avaliação e supervisão da educação superior no sistema federal de educação, e o Cadastro e-MEC de Instituições e Cursos Superiores e consolida disposições sobre indicadores de qualidade, banco de avaliadores (Basis) e o Exame Nacional 
de Desempenho de Estudantes (ENADE) e outras disposições. Brasília, DF, 2010. Diário Oficial da União, Brasília, 29 dez. 2010. Seção1. Brasília, 2010. Disponível em: http://pesquisa.in.gov.br/imprensa/jsp/visualiza/index.jsp?jornal=1\&pagina=23\&data=29/12/2 010. Acesso em: 22 mar. 2016.

BRASIL. Ministério da Educação. Instituto Nacional de Estudos e Pesquisas Educacionais Anísio Teixeira. Diretoria de Avaliação da Educação Superior. Sistema Nacional de Avaliação da Educação Superior. Instrumento de Avaliação Institucional Externa. Brasília, DF, 2014a. Disponível em: <http://download.inep.gov.br/ educacaosuperior/avaliacao_institucional/instrumentos/2014/instrumento_institucional.pdf $>$. Acesso em: 22 mar. 2016.

BRASIL. Ministério da Educação. Portaria nº 92, de 31 de janeiro de 2014. Aprova, em extrato, os indicadores do Instrumento de Avaliação Institucional Externa para os atos de credenciamento, recredenciamento e transformação de organização acadêmica, modalidade presencial, do Sistema Nacional de Avaliação da Educação Superior. Diário Oficial da União, Brasília, 4 fev. 2014. Seção 1, p. 5. Brasília, 2014b. Disponível em: $<$ http://pesquisa.in.gov.br/imprensa/jsp/visualiza/index.jsp?jornal=1\&pagina $=5 \&$ data $=04 / 02 /$ 2014>. Acesso em: 22 mar. 2016.

BRASIL. Ministério da Educação. Instituto Nacional de Estudos e Pesquisas Educacionais Anísio Teixeira. Diretoria de Avaliação da Educação Superior. Sistema Nacional de Avaliação da Educação Superior. Instrumento de avaliação de cursos de graduação presencial e à distância. Brasília, 2015.

CAVALIERI, Adriane; MACEDO-SOARES, T. Diana L. v. A.; THIOLLENT, Michel. Avaliando o desempenho da universidade. Rio de Janeiro: Ed. PUC-Rio; São Paulo: Loyola, 2004.

DIAS SOBRINHO, José. Universidade: processos de socialização e processos pedagógicos. In DIAS SOBRINHO, José; BALZAN, Newton Cesar. (Orgs.). Avaliação institucional: teoria e experiências. São Paulo: Cortez, 2005.

DIAS SOBRINHO, José. Avaliação: políticas educacionais e reformas da educação superior. São Paulo: Cortez, 2003.

DIAS SOBRINHO, José; BALZAN, Newton Cesar (Orgs.). Avaliação institucional: teoria e experiências. São Paulo: Cortez, 2005.

DIAS, Ana Maria I.; KETZER, Solange M. (Org.). Memória do ForGRAD: 20 anos do Fórum Nacional de Pró-Reitores de Graduação das Universidades Brasileiras. Unidade da Diversidade. Edição Comemorativa. Porto Alegre: EDIPUCRS, 2007.

GATTI, Bernadete A. Avaliação institucional e acompanhamento de instituições de educação superior: estudos em avaliação educacional. São Paulo: Fundação Carlos Chagas, 2000. v. 21.

RISTOFF, Dilvo I. Algumas definições de avaliação. Avaliação, Campinas, v. 8, n. 1, p. 1930, mar. 2003.

SINAES. Sistema nacional de avaliação da educação superior: da concepção à regulamentação. 5. ed. rev. e amp. Brasília: INEP, 2009. 
Enedina Betânia Leite de Lucena Pires Nunes - Universidade Federal do Tocantins Palmas | TO | Brasil. Contato: enedina@uft.edu.br

Michelle Matilde Semigueem Lima Trombini Duarte - Universidade Federal do Tocantins Palmas | TO | Brasil. Contato: msduarte@uft.edu.br

Isabel Cristina Auler Pereira - Universidade Federal do Tocantins Palmas | TO | Brasil. Contato: isabel@uft.edu.br

Artigo recebido em 5 de maio de 2016 e aprovado em 14 de julho de 2016. 